\title{
A Review of Vascular Disease Risk Factors and Multiple Sclerosis
}

\author{
Meena R Kannan and Vijayshree Yadav \\ Portland Veterans Affairs Medical Center and Oregon Health Sciences University, Portland, OR, US
}

DOI: https://doi.org/10.17925/USN.2017.13.02.90

$\mathrm{M}$ ultiple sclerosis (MS) is the most common demyelinating disease of the central nervous system and the most common non-traumatic cause of disability in young adults. Recent research shows that vascular disease risk factors (VDRFs) such as obesity, smoking, hyperlipidemia, hypertension, type II diabetes mellitus, and metabolic syndrome, can influence MS on its onset, disease activity, progression, and resultant disability. This review evaluates the current knowledge on the role of VDRFs on outcomes among people with MS (PWMS) and shows that while VDRF prevalence may or may not be higher among PWMS compared with the general population, its presence can influence MS in myriad ways. Management of VDRFs through early detection and treatment may be a promising approach to improving outcomes in PWMS.

\section{Keywords}

Multiple sclerosis, smoking, obesity, dyslipidemia, hypertension, type II diabetes, metabolic syndrome

Disclosure: No funding was received in the publication of this article. Meena Kannan and Vijayshree Yadav have no relevant conflicts of interest to declare.

Compliance with Ethics: This study involves a review of the literature and did not involve any studies with human or animal subjects performed by any of the authors.

Authorship: All named authors meet the International Committee of Medical Journal Editors (ICMJE) criteria for authorship of this manuscript, take responsibility for the integrity of the work as a whole, and have given final approval for the version to be published.

open Access: This article is published under the Creative Commons Attribution Noncommercial License, which permits any noncommercial use, distribution, adaptation, and reproduction provided the original author(s) and source are given appropriate credit.

Received: April 13, 2017

Accepted: May 16, 2017

Citation: US Neurology, 2017;13(2):90-3

Corresponding Author: Vijayshree Yadav, Mail Code L226, 3181 S.W. Sam Jackson Park Road, Portland, OR 97239, US.

E: yadavv@ohsu.edu
Multiple sclerosis (MS) is the most common inflammatory demyelinating disease of the central nervous system and the most common non-traumatic cause of disability in young adults. MS affects nearly 400,000 people in the US and 2.5 million people worldwide. While the precise etiology of MS is unknown, it is suspected that the disease causation is multifactorial, in which exposure to environmental factors triggers an autoimmune response among genetically susceptible individuals. ${ }^{1}$ Recent research suggests an important plausible role of vascular disease risk factors (VDRFs) such as smoking, obesity, hyperlipidemia, hypertension, diabetes, and metabolic syndrome on MS pathogenesis. It is estimated that nearly $50 \%$ of people with MS (PWMS) have at least one VDRF at the time of their MS diagnosis, and are more likely than healthy controls to have more than one VDRF. ${ }^{2,3}$ The presence of VDRFs among PWMS appears to be associated not only with ongoing radiographic disease activity and progression on magnetic resonance imaging (MRI), but also with increased risk of clinical disability progression..$^{3-5}$ The purpose of this paper is to examine the current literature on the relationship between these potentially modifiable factors that affect MS onset, activity, progression, and disability, and identify potential targets for improving clinical outcome among PWMS.

\section{Smoking}

The association between smoking and MS susceptibility has been investigated extensively over the past several years, with studies consistently suggesting an increased risk ranging from 27-100\% among current smokers compared with non-smokers, with several studies suggesting a dose-response relationship. ${ }^{-8}$ Passive exposure to tobacco smoke has also been associated with an increased risk of MS overall, estimated at nearly $25 \%$ compared with those not exposed to environmental tobacco smoke. $6,9,10$ The prevalence of current smoking ranges from $17-29 \%$, with one study suggesting nearly $5 \%$ of subjects started smoking after disease onset., ${ }^{3,11-13}$

Smoking among PWMS has also been associated with poor clinical and radiographic outcomes. A longitudinal study following more than 100 people with clinically isolated syndrome over 3 years showed that $75 \%$ of current smokers and only $51 \%$ of non-smokers developed clinically definite MS (CDMS), with smokers having an $80 \%$ increased risk for progression to CDMS and significantly shorter time interval to their first relapse compared with non-smokers. ${ }^{14}$ Current smokers with MS were not only more than twice as likely to progress from a relapsing to progressive phenotype but also underwent this transition earlier compared with non-smokers. ${ }^{15,16}$ Current smokers had significantly worse MS disability at baseline than never-smokers, as measured by the Expanded Disability Severity Scale (EDSS) and MS Severity Scale, as well as a $64 \%$ and $34 \%$ increased risk of reaching EDSS 4 and 6 compared with non-smokers and former smokers, respectively. Smoking among PWMS has been shown to be associated with decreased whole brain volume and increased T2 lesion volume, increased contrast enhancing lesions, and T1 lesion volume on MRI. ${ }^{17}$ 
On the other hand, individuals who stopped smoking either before or after the onset of the disease had a significantly lower risk of reaching risk of conversion from a relapsing to progressive phenotype, with similar rates between former smokers and never-smokers. Furthermore, former smokers reached the progressive state an estimated 8 years later than current smokers. ${ }^{15,16,18}$ More recent findings suggest that smoking cessation has also been associated with a significant decline in the rate of brain volume loss compared with current smokers. ${ }^{19}$ Given the high prevalence rates of current smoking among PWMS, interventions aimed at smoking cessation may substantially improve MS-related outcomes and reduce the risk of developing smoking-related comorbidities. Active counseling about smoking cessation may provide a low cost and substantially beneficial intervention among PWMS.

\section{Obesity}

Obesity has also been studied extensively as a modifiable risk factor for the onset and progression of MS. Several studies have suggested an increased risk of developing MS among overweight (body mass index [BMI] $\left.25.0-29.9 \mathrm{~kg} / \mathrm{m}^{2}\right)$ or obese $\left(\mathrm{BMI} \geq 30 \mathrm{~kg} / \mathrm{m}^{2}\right)$ individuals, particularly among overweight or obese female youth compared with male youth. This increased risk was also found to be dose-dependent, with higher BMI associated with higher risk of developing MS. ${ }^{20-24}$ Recent studies have estimated that 23-31\% of PWMS are overweight and $18-25 \%$ are obese, with the highest prevalence in North America. ${ }^{2,25,26}$ While some studies suggest that PWMS have an increased risk of becoming overweight or obese, ${ }^{27}$ others suggest a similar or decreased risk compared with healthy controls. ${ }^{3,28,29}$

Recent studies have suggested higher BMI was independently and significantly associated with increased levels of disability, but not relapse rate..$^{30,31}$ Importantly, the presence of overweight status or obesity in MS has been associated with an increase in the number of comorbidities including a nearly five-fold increase in developing diabetes or hypertension and more than two-fold increased risk of developing depression; this study also demonstrated that an increasing number of comorbidities was related to decreased quality of life and increased odds of disability. ${ }^{26}$ Overweight or obese status has also been independently associated with increased T1 lesion volume compared with those with normal BMI (18.5-24.9 kg/m²), whereas the presence of additional VDRFs has been associated with decreased whole brain and gray matter volume and increased T2 lesion volume compared with subjects without these risk factors. ${ }^{3}$ Some data also suggest that elevated BMI may also influence response to diseasemodifying treatment. Among eighty-six PWMS who were treated with interferon-beta, $80 \%$ of overweight or obese subjects had continued MRI activity compared with $48 \%$ of matched controls with normal BMI. In addition, only $13 \%$ of the overweight/obese subjects achieved no evidence of disease activity status, compared with $26 \%$ of normal-weight subjects. ${ }^{32}$ While further studies are needed to clarify this relationship, strategies targeting the overweight and obesity may provide a promising avenue for improving clinical and radiographic outcomes as well as quality of life.

\section{Dyslipidemia}

The prevalence of dyslipidemia among PWMS is estimated to be $14-49 \%$, with studies showing either higher or comparable rates to matched control populations. ${ }^{33-35}$ Studies investigating the relationship between dyslipidemia and disease activity have shown mixed findings. While dyslipidemia has not been clearly associated with MS relapse rate, recent studies have demonstrated a relationship between disability progression and dyslipidemia, specifically elevated total cholesterol, triglycerides, and decreased high-density lipoprotein (HDL). ${ }^{31,36,37}$ The North American Research Committee on MS (NARCOMS) registry showed that PWMS who developed dyslipidemia had a 83\% increased risk of developing mild visual disability, $75 \%$ increased risk of moderate visual disability, and 59\% increased risk of severe visual disability. ${ }^{38}$ These subjects also had a $35 \%$ increased risk of early gait disability, 33\% increased risk of requiring unilateral assistance, and $24 \%$ increased risk of requiring bilateral assistance compared with those without dyslipidemia. ${ }^{5}$ Subjects with elevated HDL levels were found to have deceased contrast-enhancing lesion volumes, while those with higher total cholesterol demonstrated lower whole brain volumes, when compared with matched controls. ${ }^{37}$

Six studies have investigated the use of adjunctive statin therapy with interferon-beta in the management of relapsing MS. Among the two studies comparing simvastatin to placebo, the findings were mixed with only one suggesting reduced relapse rate, and neither showing differences between groups in T2 lesions, contrast-enhancing lesions, time to first relapse, or EDSS change. ${ }^{39,40}$ The four studies comparing atorvastatin with placebo also had mixed findings with two suggesting a reduction in disease activity, one showing an increase in disease activity, and another showing no difference between groups..$^{41-44}$ Overall, further studies regarding adjuvant statin and disease-modifying therapy are needed to better understand their role in MS disease activity and progression.

These findings suggest that, while dyslipidemia may occur at similar rates among people with and without MS, high total cholesterol and triglycerides may lead to worse clinical and radiographic outcomes among PWMS, whereas higher HDL levels may provide a protective effect. At present, it is unclear whether these findings are secondary to dyslipidemia alone, or the combined effects of both dyslipidemia and MS, and therefore requires further studies to better clarify this relationship.

\section{Hypertension}

Prevalence estimates of hypertension among PWMS have varied from $23-30 \%$ in the NARCOMS registry to $11 \%$ in an international survey, with highest rates in North America at $16.7 \%{ }^{2,25,26}$ Some studies have suggested an increased prevalence of hypertension among subjects with MS compared with healthy controls, ${ }^{33,34,36}$ whereas others have shown either similar, ${ }^{5,45}$ or decreased prevalence. ${ }^{46}$

While the relationship between hypertension and relapse rate remains unclear, findings from the NARCOMS registry showed increased levels of disability among PWMS with comorbid hypertension..$^{38}$ This study demonstrated 29\% increased risk of early gait disability, 25\% increased risk of requiring unilateral assistance, and $17 \%$ increased risk of requiring bilateral assistance among those with hypertension compared with those without hypertension. ${ }^{5}$ Further analysis within this registry showed that PWMS who develop hypertension had a $32 \%$ increased risk of developing mild visual disability, $31 \%$ increased risk of moderate visual disability, and $16 \%$ increased risk of severe visual disability. ${ }^{38}$ Similarly, a recent study showed an increased risk of disability among hypertensive MS subjects, estimated at 62.3\%, 51.2\%, and 16.9\% of reaching EDSS 4, 6 , and 8 , respectively. Interestingly, this study also found that the rate of disability progression was slower when compared with individuals without hypertension. ${ }^{47}$ The presence of hypertension alone has been associated with decreased gray matter and cortical volume among PWMS compared 
with those without, whereas the presence of hypertension in addition to other VDRFs, including smoking or overweight/obesity, was also associated with increased $\mathrm{T} 2$ lesion volume and decreased whole brain and gray matter volume. ${ }^{3}$ These findings suggest that further studies are needed to better clarify the relationship between hypertension and MS-related disease activity and progression, as well as the potential role of anti-hypertensives as neuroprotective agents.

\section{Type 2 diabetes mellitus}

The prevalence of type 2 diabetes mellitus (T2DM) among PWMS was estimated at 3-8\%, with the highest prevalence in North America at 4.1\%. ${ }^{5,26,35}$ Some studies have suggested higher rates of impaired fasting glucose tolerance and diabetes among PWMS, ${ }^{34,48}$ whereas others have suggested decreased or similar rates. ${ }^{5,45}$

Nevertheless, studies have fairly consistently demonstrated worse clinical outcomes among MS subjects with T2DM compared with those without T2DM. Among subjects in the NARCOMS registry, those with comorbid T2DM had a $29 \%$ increased risk of early gait disability, $28 \%$ increased risk of requiring unilateral assistance, and $56 \%$ increased risk of requiring bilateral assistance compared with those without $\mathrm{T} 2 \mathrm{DM},{ }^{5}$ as well as a $35 \%$ increased risk of developing mild visual disability, $41 \%$ increased risk of moderate visual disability, and $54 \%$ increased risk of severe visual disability. ${ }^{38}$

While these findings do not clearly suggest an increased prevalence of T2DM among PWMS, when present, T2DM is associated with worse clinical outcomes in PWMS compared with those without T2DM. While these findings suggest a role for targeting glycemic control in improving clinical outcomes among this patient population, further studies are needed in this area.

\section{Metabolic syndrome}

Metabolic syndrome, also known as insulin resistance syndrome or syndrome $X$, is a disease state encompassing specific cardiovascular risk factors including abdominal obesity, dyslipidemia, hypertension, and abnormal glycemia. Higher body weight is a major risk factor for metabolic syndrome. ${ }^{49,50}$ While the exact definitions of metabolic syndrome may vary based upon various expert panels, metabolic syndrome often serves to identify individuals at risk for diabetes, ischemic stroke, and heart disease. ${ }^{51}$
A recent study showed metabolic syndrome prevalence among PWMS to be $30 \%$, similar to the general population, with no significant differences between groups in disease duration, degree of disability, or number of steroid treatments. ${ }^{51}$ In this study, PWMS with moderate disability (EDSS $\geq 3$ ) were included and among those, an estimated $56 \%$ had central obesity as measured by waist circumference. The study showed prevalence of treated and untreated hypertension to be $28 \%$ and $18 \%$, respectively, $36 \%$ had fasting hyperglycemia, 31\% had treated dyslipidemia, 26\% had elevated triglyceride levels, and $28 \%$ had low HDL-cholesterol. ${ }^{51}$

A recent study examined the effect of metformin or pioglitazone, drugs approved to treat T2DM, on treating metabolic syndrome and MS disease activity in obese PWMS. This study showed significantly lower systolic blood pressure and lower fasting glucose, fasting insulin, glycosylated hemoglobin, total cholesterol, low-density lipoprotein, and triglyceride levels in subjects treated with metformin or pioglitazone as compared with the untreated control group. Notably, metformin- or pioglitazone-treated subjects also showed a significantly lower number of new or enlarging T2 lesions at 6 months and contrast-enhancing lesions at 2 years for the control group, compared with the untreated control group. ${ }^{52}$ This limited, albeit important, open-label study opens a whole new avenue that can potentially help manage MS better. Further studies are needed to better understand the relationship of metabolic syndrome and MS pathogenesis.

\section{Conclusion}

MS is a disease with a poorly understood diversity of phenotypes. Emerging data suggests that presence or absence of VDRFs among PWMS may partially explain these differences in clinical outcomes. While the prevalence of VDRFs may not differ between PWMS and the general population, evidence suggests that the presence of VDRFs among PWMS plays a significant role in ongoing disease activity and progression. Just as the prevalence of VDRFS is increasing in the general population, similar trends are expected in the MS population. Early identification and management of comorbid VDRFs, including lifestyle modification and targeted medical management, may improve patient outcomes by slowing disease activity and disability progression, and reducing the risk of other comorbidities that can affect quality of life. While optimizing the management of VDRFs will not cure MS, it provides an additional promising avenue for improving overall health and quality of life in this population.
1. Belbasis $L$, Bellou V, Evangelou $E$, et al., Environmental risk factors and multiple sclerosis: an umbrella review of systematic reviews and meta-analyses, Lancet Neurol, 2015;14:263-73.

2. Marrie R, Horwitz R, Cutter G, et al., High frequency of adverse health behaviors in multiple sclerosis, Mult Scler, 2009;15:105-13.

3. Kappus N, Weinstock-Guttman B, Hagemeier J, et al., Cardiovascular risk factors are associated with increased lesion burden and brain atrophy in multiple sclerosis, J Neurol Neurosurg Psychiatry, 2016;87:181-7.

4. Moccia M, Lanzillo R, Palladino R, et al., The Framingham cardiovascular risk score in multiple sclerosis, Eur J Neurol, 2015;22:1176-83.

5. Marrie RA, Rudick R, Horwitz R, et al., Vascular comorbidity is associated with more rapid disability progression in multiple sclerosis, Neurology, 2010;74:1041-7.

6. Hedström AK, Hillert J, Olsson T, Alfredsson L, Smoking and multiple sclerosis susceptibility, Eur J Epidemiol, 2013;28:867-74.

7. O'Gorman C, Bukhari W, Todd A, et al., Smoking increases the risk of multiple sclerosis in Queensland, Australia, J Clin Neurosci, 2014;21:1730-3.

8. Hernan MA, Olek MJ, Ascherio A, Cigarette smoking and incidence of multiple sclerosis, Am J Epidemiol, 2001;154:69-74.

9. Hedström AK, Bomfim IL, Barcellos LF, et al., Interaction between passive smoking and two HLA genes with regard to multiple sclerosis risk, Int J Epidemiol, 2014;43:1791-8.

10. Ramagopalan SV, Lee JD, Yee IM, et al., Association of smoking with risk of multiple sclerosis: a population-based study, J Neurol, 2013;260:1778-81.

11. Turner AP, Kivlahan DR, Kazis LE, Haselkorn JK, Smoking among veterans with multiple sclerosis: prevalence correlates, quit attempts, and unmet need for services, Arch Phys Med Rehabil, 2007:88:1394-9.

12. McKay KA, Tremlett H, Fisk JD, et al., Adverse health behaviours are associated with depression and anxiety in multiple sclerosis: a prospective multisite study, Mult Scler, 2016;22:685-93.

13. Hedström AK, Baarnhielm M, Olsson T, Alfredsson L, Tobacco smoking, but not Swedish snuff use, increases the risk of multiple sclerosis, Neurology, 2009:73:696-701.

14. Di Pauli F, Reindl $M$, Ehling $R$, et al., Smoking is a risk factor for early conversion to clinically definite multiple sclerosis, Mult Scler 2008;14:1026-30.

15. Healy BC, Ali EN, Guttmann CR, et al., Smoking and disease progression in multiple sclerosis, Arch Neurol, 2009;66:858-64.

16. Ramanujam R, Hedström AK, Manouchehrinia A, et al., Effect of smoking cessation on multiple sclerosis prognosis, JAMA Neurology, 2015;72:1117-23.

17. Zivadinov R, Weinstock-Guttman B, Hashmi K, et al., Smoking is associated with increased lesion volumes and brain atrophy in multiple sclerosis, Neurology, 2009;73:504-10.

18. Manouchehrinia A, Tench $C R$, Maxted J, et al., Tobacco smoking and disability progression in multiple sclerosis: United Kingdom cohort study, Brain, 2013;136:2298-304.
19. Seraji-Bozoergzad N, Bao F, Razmjou S, et al., Smoking cessation slows brain volume loss in multiple sclerosis, Presented at: European Committee for Treatment and Research in Multiple Sclerosis (ECTRIMS) Congress, Barcelona, Spain, October 9, 2015. Abstr. 116335.

20. Munger KL, Bentzen J, Laursen B, et al., Childhood body mass index and multiple sclerosis risk: a long-term cohort study, Mult Scler, 2013;19:1323-9.

21. Langer-Gould A, Brara SM, Beaber BE, Koebnick C, Childhood obesity and risk of pediatric multiple sclerosis and clinically isolated syndrome, Neurology, 2013;80:548-52.

22. Hedström AK, Olsson T, Alfredsson L, High body mass index before age 20 is associated with increased risk for multiple sclerosis in both men and women, Mult Scler, 2012;18:1334-6.

23. Munger KL, Chitnis T, Ascherio A, Body size and risk of MS in two cohorts of US women, Neurology, 2009;73:1543-50.

24. Kavak $\mathrm{KS}$, Teter $\mathrm{BE}$, Hagemeier J, et al., Higher weight in adolescence and young adulthood is associated with an earlier age at multiple sclerosis onset, Mult Scler, 2015;21:858-65.

25. Marrie RA, Horwitz Rl, Cutter G, et al., Association between comorbidity and clinical characteristics of MS, Acta Neurologica Scandinavica, 2011;124:135-41.

26. Marck $\mathrm{CH}$, Neate $\mathrm{SL}$, Taylor $\mathrm{KL}$, et al., Prevalence of comorbidities, overweight and obesity in an International sample of people with multiple sclerosis and associations with modifiable lifestyle factors, PLoS One, 2016;11:e0148573. 
27. Khurana SR, Bamer AM, Turner AP, et al., The prevalence of overweight and obesity in veterans with multiple sclerosis, $A m$ J Phys Med Rehabil, 2009;88:83-91.

28. Allen NB, Lichtman JH, Cohen HW, et al., Vascular disease among hospitalized multiple sclerosis patients, Neuroepidemiology, 2008;30:234-8.

29. Sioka C, Fotopoulos A, Georgiou A, et al., Body composition in ambulatory patients with multiple sclerosis, J Clin Densitom, 2011;14:465-70.

30. Tettey P, Simpson S Jr., Taylor B, et al., An adverse lipid profile is associated with disability and progression in disability, in people with MS, Mult Scler, 2014;20:1737-44.

31. Tettey P, Simpson S Jr., Taylor B, et al., Adverse lipid profile is not associated with relapse risk in MS: results from an observationa cohort study, J Neurol Sci, 2014:340:230-2.

32. Kvistad SS, Myhr KM, Holmoy T, et al., Body mass index influence interferon-beta treatment response in multiple sclerosis, J Neuroimmunol, 2015;288:92-7.

33. Lavela SL, Prohaska TR, Furner S, Weaver FM, Chronic diseases in male veterans with multiple sclerosis, Prev Chronic Dis, 2012;9:E55

34. Kang JH, Chen $\mathrm{YH}$, Lin HC, Comorbidities amongst patients with multiple sclerosis: a population-based controlled study, Eur J Neurol, 2010;17:1215-9.

35. Marrie RA, Yu BN, Leung S, et al., Rising prevalence of vascular comorbidities in multiple sclerosis: validation of administrative definitions for diabetes, hypertension, and hyperlipidemia, Mult Scler, 2012;18:1310-9.
36. Tettey P, Siejka D, Simpson S Jr., et al., Frequency of comorbidities and their association with clinical disability and relapse in multiple sclerosis, Neuroepidemiology, 2016;46:106-13.

37. Weinstock-Guttman B, Zivadinov R, Mahfooz N, et al., Serum lipid profiles are associated with disability and MRI outcomes in multiple sclerosis, J Neuroinflamm, 2011;8:127.

38. Marrie RA, Cutter G, Tyry T, Substantial adverse association of visual and vascular comorbidities on visual disability in multiple sclerosis, Mult Scler, 2011;17:1464-71.

39. Sorensen PS, Lycke J, Eralinna JP, et al., Simvastatin as add-on therapy to interferon beta-1a for relapsing-remitting multiple sclerosis (SIMCOMBIN study): a placebo-controlled randomised phase 4 trial, Lancet Neurol, 2011;10:691-701.

40. Togha M, Karvigh SA, Nabavi M, et al., Simvastatin treatment in patients with relapsing-remitting multiple sclerosis receiving interferon beta 1a: a double-blind randomized controlled trial, Mult Scler, 2010;16:848-54.

41. Paul F, Waiczies $S$, Wuerfel J, et al., Oral high-dose atorvastatin treatment in relapsing-remitting multiple sclerosis, PLOS One, 2008:3:e1928.

42. Birnbaum G, Cree B, Altafullah I, et al., Combining beta interferon and atorvastatin may increase disease activity in multiple sclerosis, Neurology, 2008:71:1390-5.

43. Kamm CP, El-Koussy M, Humpert $\mathrm{S}$, et al., Atorvastatin added to interferon beta for relapsing multiple sclerosis: a randomized controlled trial, J Neurol, 2012;259:2401-13.

44. Lanzillo R, Orefice G, Quarantelli M, et al., Atorvastatin combined to interferon to verify the efficacy (ACTIVE) in relapsing-remitting active multiple sclerosis patients: a longitudinal controlled trial o combination therapy, Mult Scler, 2010;16:450-4.

45. Sternberg Z, Leung C, Sternberg D, et al., The prevalence of the classical and non-classical cardiovascular risk factors in multiple sclerosis patients, CNS Neurol Disord Drug Targets, 2013;12:104-11.

46. Simpson RJ, McLean G, Guthrie B, et al., Physical and mental health comorbidity is common in people with multiple sclerosis: nationally representative cross-sectional population database analysis, BMC Neurol, 2014;14:128

47. Dagan A, Gringouz I, Kliers I, Segal G, Disability progression in multiple sclerosis is affected by the emergence of comorbid arterial hypertension, I Clin Neurol, 2016;12:345-50.

48. Wens I, Dalgas U, Deckx N, et al., Does multiple sclerosis affect glucose tolerance?, Mult Scler, 2013;20:1273-6.

49. Grundy SM, Cleeman Jl, Daniels SR, et al., Diagnosis and management of the metabolic syndrome: an American Heart Association/National Heart, Lung, and Blood Institute scientific statement, Circulation, 2005;112:2735-52

50. Alberti KGM, Zimmet P, Shaw J, for the IDF Epidemiology Task Force Consensus Group, The metabolic syndrome - a new worldwide definition, Lancet, 2005:366:1059-62.

51. Pinhas-Hamiel O, Livne M, Harari G, Achiron A, Prevalence of overweight, obesity and metabolic syndrome components in multiple sclerosis patients with significant disability, Eur J Neurol, 2015:22:1275-9.

52. Negrotto L, Farez MF, Correale J, Immunologic effects of metformin and pioglitazone treatment on metabolic syndrome and multiple sclerosis, JAMA Neurol, 2016;73:520-8. 